\title{
An evaluation of the use of pooled samples in studies of genetic variation
}

\author{
THOMAS KRAFT* \& TORBJÖRN SÄLL \\ Department of Genetics, University of Lund, Solvegatan 29, S-223 62 Lund, Sweden
}

\begin{abstract}
When using molecular markers to study genetic variation, either the sampled individuals can be analysed individually or the individuals can be pooled and only the pools analysed (pooled samples). A theoretical investigation was carried out into the use of pooled samples in the detection of alleles and providing maximum likelihood estimates of allele frequency. The results show that, in many cases, pooled samples are more efficient than samples of individuals. Of the different pool sizes studied, small pools containing two or three individuals showed the smallest expected squared error of allele frequency estimates.
\end{abstract}

Keywords: allele frequencies, genetic variation, maximum likelihood, pooled samples.

\section{Introduction}

Genetic variation is of fundamental importance in many areas of basic and applied biology. It is a prerequisite for evolution, both in natural systems and in the process of breeding crops and domestic animals, as well as being the basis for genetic fingerprinting. Accordingly, there has been an interest in studying genetic variation in arbitrary populations and species. This has only been possible in recent decades through the introduction of different marker techniques. The development of such DNA-based marker systems as restriction fragment length polymorphism (RFLP), random amplified polymorphic DNA (RAPD), AFLP and microsatellites, in particular, has made it possible to access the pattern of variation in virtually any organism. A daunting aspect of such investigations, on the other hand, has been the large number of individuals it has been necessary to investigate in order to obtain adequate estimates of different population parameters. Because many DNAbased marker systems are quite expensive, the total cost of any such project limits the number of accessions that can be screened.

One approach that might possibly increase the amount of information obtained for a given amount of time and money invested is to pool individuals and then analyse these pools, which are termed bulked or pooled samples. Although such a strategy necessarily means information on single individuals being lost, this may be compensated for, in some cases, by a greater

*Correspondence and present address: Novartis Seeds AB, Box 302, S-261 23 Landskrona, Sweden.

E-mail: thomas.kraft@seeds.novartis.com accuracy in the estimates of various population parameters. Use of pooled samples has been evaluated and found useful in many different applications. Churchill et al. (1993) studied the value of pooled samples for the high-resolution mapping of DNA markers. Darvasi \& Soller (1994) recommended the use of pooled samples in the mapping of QTLs, using a method they termed selective DNA pooling. The merits of pooled samples in detecting and describing genetic variation have been studied using isozymes (Nemec \& Stary, 1984), RAPDs (Yu \& Pauls, 1993; Sweeney \& Danneberger, 1994; Kongkiatngam et al., 1996), RFLPs (Kidwell et al., 1994) and microsatellites (LeDuc et al., 1995). The last investigations compared the levels of variation found in groups of individuals when studied as pooled samples and when studied individually. Kraft et al. (1997) used pooled samples in an RFLP study of genetic variation in sugar beet and also used simulations to evaluate the use of pooled samples. It is difficult, however, to draw any general conclusions about pooled samples from these studies, because the use of the method has been evaluated in only very specific situations.

It should be noted that estimating allele frequencies in populations of polyploid taxa is very similar to the use of pooled samples for estimating allele frequencies in diploid populations. A DNA sample from a tetraploid individual, for example, can be viewed as a pooled sample of two diploid individuals. In electrophoretic surveys of genetic variation in polyploid organisms, an attempt is often made to estimate the number of copies of different alleles in each individual. If this is not possible, the maximum likelihood estimator of allele frequencies is the same as the estimator for pooled samples presented here. 
We have attempted to evaluate the use of pooled samples in studies of genetic variation in a more general way. When the number of copies of different alleles in each pool can be quantified accurately, through using software for image analysis, for example, the use of pooled samples is fairly straightforward. In the present study, we examine instead the worst possible case, that is of it only being possible to score alleles as being present or absent in the pools. Because the number of different alleles observed in a sample and the estimates of allele frequencies form the basis for many parameter estimators in population genetics, we analysed the efficiency of pooled samples in finding as many of the alleles in a population as possible and in estimating allele frequencies.

\section{Methods}

\section{Model}

We consider a sexual, random-mating population of diploid individuals for which genetic variation in the population is to be analysed using a co-dominant marker system such as RFLP or microsatellites. We assume that the size of the sample that can be analysed in an investigation is limited by either monetary or time considerations, and we therefore keep the sample size $b$ fixed. The random sample can consist either of $b$ individuals or of $b$ pools. When the samples consist of pools, the number of individuals in each pool is denoted by $n$. The total number of different alleles observed in a sample is designated as $a$. When the sample consists of pools, these alleles are represented by $x_{1}, \ldots, x_{a}$ gene copies in the respective pools, such that $\sum_{i} x_{\mathrm{i}}=2 n$. The values of $x_{1}, \ldots, x_{a}$, however, are unknown, alleles only being scored as either present or absent in a pool, there thus being no quantitative assessment within the pools. Also, if many individuals are contained in a pool, an allele represented by a single gene copy may not be visible. Accordingly, we introduce $p_{\min }$, which is the minimum relative frequency that an allele must have in a pool to be visible, and $x_{\min }$, which is the minimum number of gene copies required for an allele to be visible. If a pool is of limited size, so that an allele represented in the pool by a single gene copy can be detected, i.e. if $2 n p_{\min } \leq 1$, then $x_{\min }=1$.

\section{Estimating allele frequencies}

If the sample consists of individuals, then the maximum likelihood estimate of the allele frequency in the population $\hat{p}$ is the frequency of the allele in the sample (Weir, 1996). This estimate is unbiased, its variance in samples obtained from randomly mating populations being given by $V(\hat{p})=p\left(\begin{array}{ll}1 & p\end{array}\right) / 2 b$, where $b$ is the sample size (Weir, 1996). When the sample consists of pools, it is more complicated to estimate allele frequencies, because one only has observations then of the presence or absence of different alleles within the pools. Under the assumptions given in the model, the overall likelihood function for observing a given pattern of the presence or absence of a particular allele within $b$ pools will be $L=\prod_{i=1}^{b} L_{i}$, where $L_{i}$ is the likelihood function for the $i$ th pool. This function has the form $L_{i}=\sum_{x_{1}} \ldots \sum_{x_{a}}\left(2 n ! /\left(x_{1} ! \ldots x_{a} !\right)\right) p_{1}^{x_{1}} \ldots p_{a}^{x_{a}}$, where $x_{j}=$ $0, \ldots,\left(x_{\min }-1\right)$ if the $j$ th allele is not observed in the $i$ th pool, and $x_{j}=x_{\min }, \ldots, 2 n \quad \sum_{k \neq j} x_{k}$ if the $j$ th allele is observed in the pool. The summations in the likelihood function are constrained by the requirement $\sum_{j=1}^{a} x_{j}=2 n$. Maximum likelihood estimates of allele frequencies $\left(\hat{p}_{1}, \ldots, \hat{p}_{a}\right)$ for an observed distribution of presence or absence of alleles among $b$ pools are found by maximizing the likelihood function with respect to $p_{1}, \ldots, p_{a}$. The expectations for these estimates can be calculated for specific situations as $\mathrm{E}(\hat{p})=\sum_{i} \operatorname{Pr}\left(X_{i}\right) \hat{p}_{i}$, where $X_{i}$ are all possible patterns of the presence or absence of alleles within the pools and $\hat{p}_{i}$ is the allele frequency estimate corresponding to the $i$ th outcome. Similarly, the squared errors can be calculated as $\mathrm{SE}(\hat{p})=\sum_{i} \operatorname{Pr}\left(X_{i}\right)\left(\hat{p}_{i} \quad p\right)^{2}$, where $\hat{p}_{i}$ is the allele frequency estimate corresponding to $X_{i}$ and $p$ is the true allele frequency.

The calculations of expectations and squared errors for estimates of allele frequencies based on pooled samples are not very handy when there are more than two alleles or more than five pools in the samples, because the number of possible outcomes increases rapidly. Such situations were evaluated here instead by simulations. Random gene copies were drawn from populations with fixed allele frequencies to form pooled samples, maximum likelihood estimates of allele frequencies being calculated for these pools. These estimates were compared with the correct allele frequencies in the simulated population, mean squared errors being calculated by repeating this procedure 1000 times.

\section{Results and discussion}

\section{Probability of finding different alleles}

One important aspect of a population analysis is the detection of rare alleles. Despite their not contributing much to genetic variation when measured in terms of expected heterozygosity, the presence of such alleles can be of considerable theoretical and practical importance. Given the terminology applied above, the probability of finding a specific allele in a single pool is 
$P_{f}=1 \quad \sum_{x=0}^{x_{\min } 1}\left(\begin{array}{c}2 n \\ x\end{array}\right) p^{x}\left(\begin{array}{ll}1 & p)^{2 n} x\end{array}\right.$

where $p$ is the frequency of the allele in the population investigated, and $2 n$ is the number of gene copies in the pool. If a single copy of the allele is visible in the pool, i.e. if $x_{\min }=1$, the formula reduces to $P_{f}=1-(1-p)^{2 n}$. The first issue to be investigated is what pool size relative to $x_{\min }$ is optimal, i.e. whether it is optimal to use a pool size for which $x_{\min }=1$ or to use a larger pool. To investigate this, we first introduce $m=1 / p_{\min }$, i.e. the maximum size of a sample of gene copies for which a single copy of a specific allele is detectable. In a pool of $\mathrm{km}$ gene copies, $k$ copies are required for the allele to be detected and, in a pool of $k m+d$ gene copies, where $0<d<m, k+1$ copies are required for detection. It is obvious then that $P_{f}$ is maximized when the pool size is a multiple of $m$. We compared pools of size $m, 2 m$ and $3 m$ for three different values of $p_{\min }$ (Fig. 1). For all values of $p_{\min }$, the pool of size $m$ had the highest probability of alleles with a frequency $<p_{\min }$ being found, whereas the opposite is true for alleles with a frequency $>p_{\min }$. If the aim of a study is to find all the common alleles in a population, it is desirable to have pools that are as large as practically possible. For all pool sizes, however, the probability of finding common alleles is rather high, especially if samples of several pools are used. Therefore, if one wishes to find as many alleles as possible of those present in the population, it is most efficient to use pools of size $m$.

Because the most efficient pools are those in which all the alleles are detected, it is simple to compare pools with samples of individuals. The only important parameter other than allele frequency is the total number of genes sampled, i.e. $2 n b$. The overall formula for the probability of finding an allele then is $P_{f}=1-(1-p)^{2 n b}$. This formula has well-known properties. In Fig. 2, the relation between $P_{f}$ and $p$ is shown for different values of $2 n b$. Clearly, pooled samples are very efficient for finding as many different alleles as possible. For example, if $p_{\min }=0.10$, the probability of finding a rare allele among five individuals will obviously be the same regardless of whether they are analysed individually or as a pool.

\section{Estimates of allele frequencies - simple cases}

The expected values of estimates of allele frequencies based on pooled samples depend on many different parameters, specifically on the number of individuals in each pool $(n)$, the number of pools in the sample $(b)$, the (a)

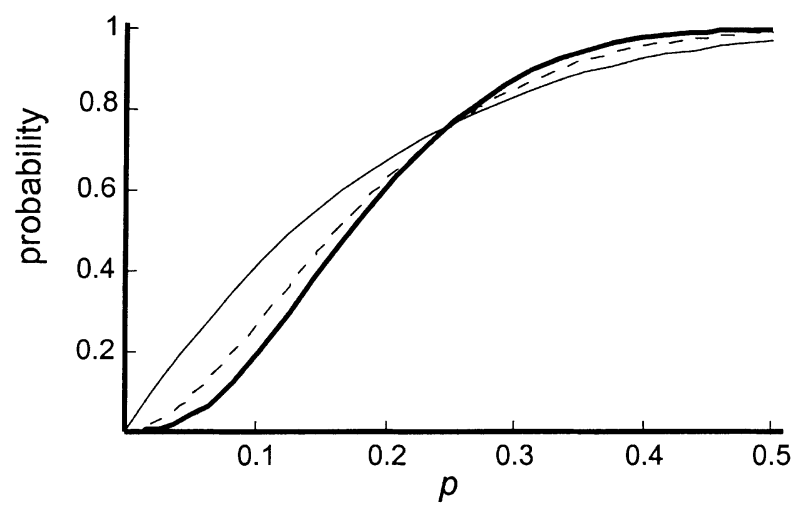

(b)

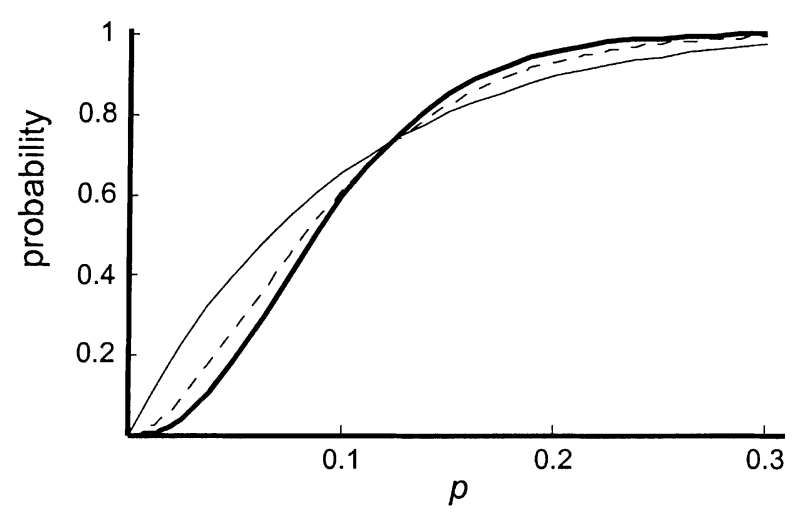

(c)

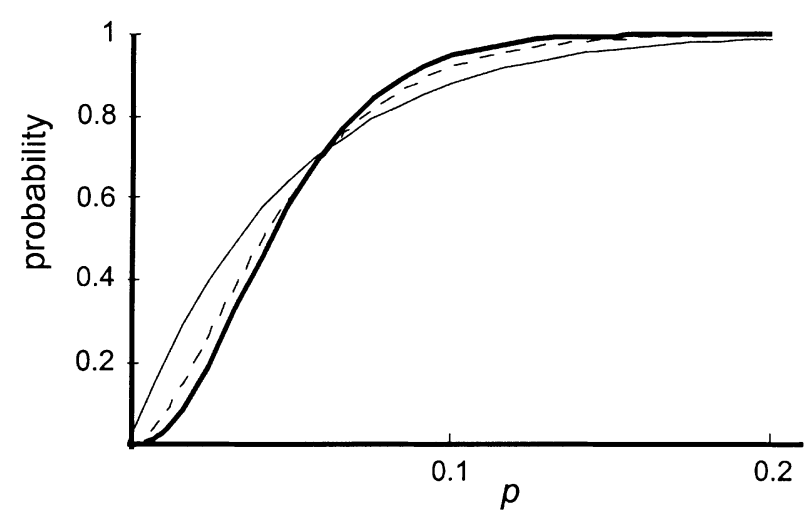

Fig. 1 Relationship between the frequency of an allele and the probability of detecting it in a single gene pool. The numbers of gene copies in a pool were $1 / p_{\min }$ (solid line), $2 / p_{\min }$ (dashed line) and $3 / p_{\min }$ (thick line). (a) $p_{\min }=0.20$,

(b) $p_{\min }=0.10$, (c) $p_{\min }=0.05$. Note the differing scales on the abscissa.

detection level $\left(p_{\min }\right)$ and the allele frequencies and inbreeding coefficients in the population sampled. It would be highly impractical to study all possible 


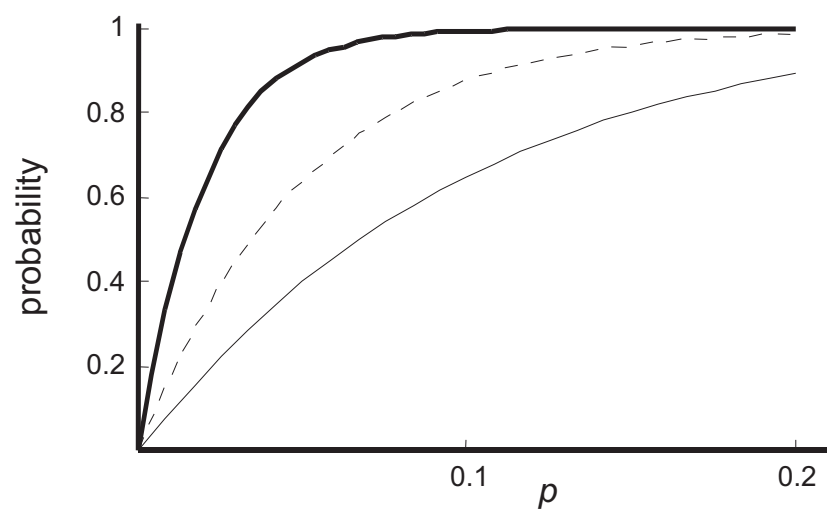

Fig. 2 Relationship between the frequency of an allele and the probability of detecting it. The total numbers of gene copies analysed were 10 (solid line), 20 (dashed line) and 50 (thick line). (As long as the size of a single pool is not larger than $1 /$ $p_{\text {min }}$, the results will be the same, regardless of whether the gene copies are analysed as samples of individuals or as samples of one or several pools.)

combinations of these parameters. Let us start, therefore, by analysing some simple cases in which $b=5$, $p_{\min }=0.10$, only two different alleles are present and the populations are assumed to mate randomly. Two parameters, the allele frequencies, $p$, and the pool sizes, $n$, are allowed to vary. The relationship between the true allele frequencies and the expected allele frequency estimates for several pool sizes is shown in Fig. 3. The results show clearly that estimates based on pooled samples are biased and that the bias increases with an increase in the number of individuals in the pools. When there are only two alleles in the population, with frequencies of $0.2-0.8$, large pools almost always detect both alleles, the estimates thus being close to 0.5. The frequencies of rarer alleles, on the other hand, are underestimated in larger pools, because the frequencies of rare alleles in a particular pool will only rarely exceed $p_{\min }$, so that the estimates are close to zero. We endeavoured to improve the allele frequency estimates using the jackknife method, which is often known to reduce bias. Although, in our case, the jackknife estimates were sometimes slightly less biased than the standard estimates, the bias of the jackknife estimates was higher at other times. In addition, in some cases, the jackknife estimates were either less than zero or more than one. Accordingly, the jackknife method does not appear to be effective in the present situation. Thus, judging from the bias only, samples of individuals clearly seem to be better than pools, the former resulting in unbiased estimates. When estimates are evaluated in terms of expected squared errors, which include both the bias and the variance of the estimates, however, a

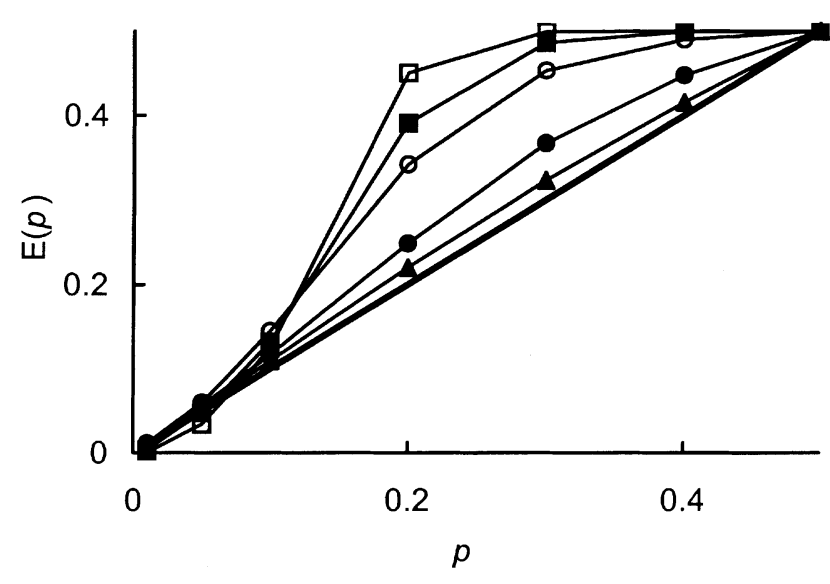

Fig. 3 Relationship between population frequency, $p$, and expectations of estimates of allele frequencies, $\mathrm{E}(p)$, for different pool sizes, those of $n=2$ (triangles), $n=3$ (filled circles), $n=5$ (open circles), $n=10$ (filled squares) and $n=20$ (open squares). The thick line represents unbiased estimates. It is assumed that only two different alleles are present, $p_{\min }=0.10$ and that each sample consists of five pools. Estimates are only shown for one of the alleles, because the estimate for the second allele is the complement of the estimate for the first allele.

different picture emerges (Fig. 4). The expected squared errors are then smaller for estimates based on small pools than for those based on individuals, probably because the estimates based on pooled samples have smaller variances. It seems very reasonable for the variance of the pooled samples to be smaller, as a greater number of individuals are then included in the sample. For all tested allele frequencies, pools consisting of two individuals were the only ones for which the expected squared errors were always found to be smaller than the variances of the individuals.

\section{Estimates of allele frequencies - general cases}

The results presented above are for comparisons between estimates based on either five pools or five individuals. These assumptions will now be relaxed by varying sample size and the number of alleles in the population. Because, in all the situations studied so far, it is the small pools $(n<5)$ that had the smallest expected squared errors, we will concentrate on pools with two to five individuals per pool.

The expected squared errors were calculated for different sample sizes, $b$, of pools and were then compared with the expected squared errors for the same sample size of individuals. For $b>5$, simulations were used to estimate the expected squared errors of estimates based on pooled samples. In most of the cases studied, 


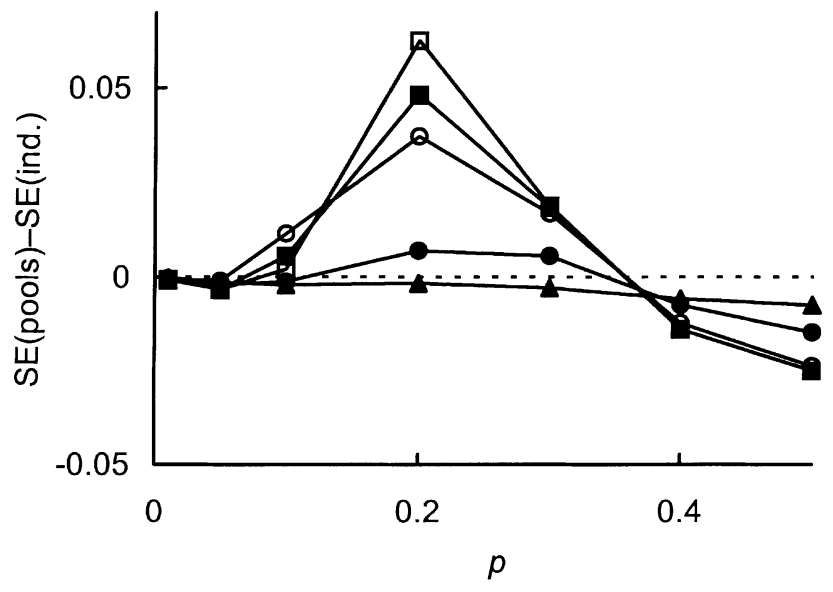

Fig. 4 Differences between samples of pools and individuals in terms of expected squared errors of estimates of allele frequencies for different population allele frequencies, $p$, and for different pool sizes, $n=2$ (triangles), $n=3$ (filled circles), $n=5$ (open circles), $n=10$ (filled squares) and $n=20$ (open squares). Sample size is five in all cases. It is assumed that only two different alleles are present and that $p_{\text {min }}=0.10$. The expected squared errors for the second allele are the same as for the first allele.

the expected squared errors were smaller for pools than for individuals (Table 1).

When more than two different alleles were modelled per population, the expected values for estimates based on pooled samples were calculated by simulations. The number of possible combinations of allele frequencies increases dramatically when the number of alleles increases to more than two. We chose to investigate only two combinations, one with a strongly skewed distribution of allele frequencies and the other with a more even distribution. The pools of two individuals performed very well and always had mean squared errors of allele frequency estimates that were smaller than the expected squared errors of estimates based on the same number of individuals instead of on pools (Table 2). Because other sets of allele frequencies studied gave similar results (data not shown), it appears that pooled samples also work well when many different alleles are present. In general, therefore, it seems best to use pools of two or three individuals.

\section{Detection level}

The minimum frequency needed for an allele to be visible, $p_{\min }$, can vary from one situation to another and must be determined empirically. Investigations in our laboratory have shown $p_{\min }$ to be $\approx 10 \%$, which is what we have used in our evaluation of pooled samples. Using instead a $p_{\min }$ of $5 \%$ or of $15 \%$ yielded similar results (data not shown). The pools of two or three individuals were not affected by the change in $p_{\min }$, because all the copies are visible in such small pools. Such pools always performed better than larger pools. Larger pools, on the other hand, can be affected greatly by a change in $p_{\min }$. As long as all the gene copies in a pool are detected, the bias in the estimates will increase when the number of individuals in the pool is increased (Fig. 3). When the pool size is increased above the level at which a single copy of an allele can no longer be detected, the bias decreases. Further increasing the pool size increases the bias, until two copies of an allele are no longer detected. At such a level, the bias then decreases. However, in pools of two or three individuals the expected squared error is always smaller still. In addition, because of small variations in experimental conditions, an allele will sometimes be detected in a pool even though its frequency in the pool is not above $p_{\min }$. Because the bias in such cases increases, it is not advisable, in practice, to use pool sizes that are close to the breakpoint at which an additional gene copy is needed to detect an allele.

Table 1 Expected squared errors of estimates of allele frequencies $(\hat{p})$ for several different sample sizes $(b)$ and for pool sizes of 2, 3, 4 and 5 individuals per pool

\begin{tabular}{|c|c|c|c|c|c|c|c|c|c|c|}
\hline \multirow[b]{2}{*}{$b$} & \multicolumn{5}{|c|}{$p=0.2$} & \multicolumn{5}{|c|}{$p=0.4$} \\
\hline & $n=2$ & $n=3$ & $n=4$ & $n=5$ & Individual & $n=2$ & $n=3$ & $n=4$ & $n=5$ & Individual \\
\hline 2 & 0.0396 & 0.0550 & 0.0673 & 0.0756 & 0.0400 & 0.0313 & 0.0187 & 0.0134 & 0.0113 & 0.0600 \\
\hline 3 & 0.0257 & 0.0402 & 0.0555 & 0.0672 & 0.0267 & 0.0255 & 0.0182 & 0.0136 & 0.0114 & 0.0400 \\
\hline 4 & 0.0184 & 0.0300 & 0.0459 & 0.0598 & 0.0200 & 0.0213 & 0.0174 & 0.0137 & 0.0116 & 0.0300 \\
\hline 5 & 0.0142 & 0.0230 & 0.0382 & 0.0532 & 0.0160 & 0.0181 & 0.0165 & 0.0136 & 0.0117 & 0.0240 \\
\hline 10 & 0.0066 & 0.0087 & 0.0171 & 0.0298 & 0.0080 & 0.0104 & 0.0129 & 0.0136 & 0.0123 & 0.0120 \\
\hline 30 & 0.0020 & 0.0021 & 0.0028 & 0.0050 & 0.0027 & 0.0036 & 0.0060 & 0.0093 & 0.0109 & 0.0040 \\
\hline 50 & 0.0012 & 0.0011 & 0.0012 & 0.0018 & 0.0016 & 0.0023 & 0.0036 & 0.0064 & 0.0096 & 0.0024 \\
\hline
\end{tabular}

Calculations are made for true allele frequencies of 0.2 and 0.4 , assuming only two alleles to be present in the population. Values in bold are for situations in which the pools result in expected squared errors as low as in the samples of individuals or lower still. 
Table 2 Expected squared errors for estimates of allele frequency $(\hat{p})$ based on samples of five individuals or on five pools with 2, 3, 4 or 5 individuals per pool

\begin{tabular}{lllllc}
\hline & $n=2$ & $n=3$ & $n=4$ & $n=5$ & Individual \\
\hline $\mathrm{p}_{1}=0.05$ & $\mathbf{0 . 0 0 3 3}$ & $\mathbf{0 . 0 0 2 6}$ & $\mathbf{0 . 0 0 3 3}$ & $\mathbf{0 . 0 0 3 7}$ & 0.0048 \\
$\mathrm{p}_{2}=0.15$ & $\mathbf{0 . 0 1 0 5}$ & 0.0133 & 0.0216 & 0.0294 & 0.0128 \\
$\mathrm{p}_{3}=0.8$ & $\mathbf{0 . 0 1 2 8}$ & $\mathbf{0 . 0 1 5 2}$ & 0.0257 & 0.0374 & 0.0160 \\
$\mathrm{p}_{1}=0.1$ & $\mathbf{0 . 0 0 6 2}$ & $\mathbf{0 . 0 0 5 7}$ & $\mathbf{0 . 0 0 6 7}$ & 0.0097 & 0.0090 \\
$\mathrm{p}_{2}=0.3$ & $\mathbf{0 . 0 1 5 2}$ & $\mathbf{0 . 0 1 8 5}$ & $\mathbf{0 . 0 1 9 8}$ & $\mathbf{0 . 0 1 8 0}$ & 0.0210 \\
$\mathrm{p}_{3}=0.6$ & $\mathbf{0 . 0 1 6 5}$ & $\mathbf{0 . 0 2 1 6}$ & 0.0254 & 0.0297 & 0.0240 \\
\hline
\end{tabular}

The expected squared errors of the estimates based on pooled samples were calculated for 1000 repeats of a simulation performed for two different sets of true allele frequencies. Values in bold are for situations in which the pools result in expected squared errors as low as in the samples of individuals or lower still.

\section{Inbreeding}

The maximum likelihood estimator used in this study is based on the assumption of random mating. If inbreeding has occurred in the population studied, so that there is an excess of homozygotes, this will be very difficult to detect in a sample of pools. Therefore, we used simulations to investigate the effect of inbreeding on our estimator. As populations become more inbred, the expected squared errors of estimates of allele frequencies based on samples of individuals tend to increase (e.g. Weir, 1996). This was also often found to be true for samples of small pools, the larger pools being affected much less (data not shown). The increase in expected squared error is caused by inbreeding effectively reducing the number of independent gene copies in the pool. With complete inbreeding, each individual in the pool represents only a single randomly chosen gene copy instead of two, as in a randomly mating population. In the case of complete inbreeding, the optimal pool size should thus be twice as large as for randomly mating populations. It is possible, therefore, to compensate for the increase in mean squared errors in inbred populations by using larger pools. Our allele frequency estimator is based on the assumption of random mating. If one has prior knowledge of the inbreeding level in the population, one can adjust the likelihood function by including this information, allowing better estimates of allele frequencies to be obtained. This correction has a rather small impact on the estimates, however, even when inbreeding is complete (data not shown).

\section{Applicability of results}

Estimates of allele frequencies and the numbers of observed alleles in a sample are often used to estimate other population parameters. Estimates of these other parameters will of course be affected by the accuracy of the allele frequency estimates, for example. We also investigated the efficiency of pooled samples in the estimation of one population parameter that is commonly used, that of expected heterozygosity, $H=1-\sum p^{2}$. The results were very similar to those for the allele frequency estimates. Once again, for all the situations investigated, pools of two or three individuals had smaller expected squared errors than samples of individuals (data not shown). It seems reasonable to assume that this is also true for most other estimates of population parameters that are based on allele frequency estimates, such as those of genetic distances between populations.

\section{Acknowledgements}

This study was made possible by a grant from the Nilsson-Ehle foundation to T.S.

\section{References}

CHURChill, G. A., GIOVANNONI, J. J. AND TANKSLEY, S. S. 1993. Pooled-sampling makes high-resolution mapping practical with DNA markers. Proc. Natl. Acad. Sci. U.S.A., 90, 16-20.

DARVASI, A. AND SOLLER, M. 1994. Selective DNA pooling for determination of linkage between a molecular marker and a quantitative trait locus. Genetics, 138, 1365-1373.

KIDWELL, K. K., AUSTIN, D. F. AND OSBORN, T. C. 1994. RFLP evaluation of nine Medicago accessions representing the original germplasm sources for North American alfalfa cultivars. Crop Sci., 34, 230-236.

KONGKIATNGAM, P., WATERWAY, M. J., COULMAN, B. E. AND FORTIN, M. G. 1996. Genetic variation among cultivars of red clover (Trifolium pratense L.) detected by RAPD markers amplified from bulk genomic DNA. Euphytica, 89, 355-361.

KRAFT, T., FRIDLUND, B., HJERDIN, A., SÄLL, T., TUVESSON, S. AND HALLDÉN, C. 1997. Estimating genetic variation in cultivated and wild beets using pools of individuals. Genome, 40, 527-533. 
LEDUC, C., MILlER, P., LICHTER, J. AND PARRY, P. 1995. Batched analysis of genotypes. PCR Methods Applications, 4, 331-336. NEMEC, V. AND STARY, P. 1984. Utilisation of isozyme analysis in the research on population diversity of aphid parasitoids (Hym., Aphidiidae). Z. Ent., 98, 150-159.

SWEENEY, P. M. AND DANNEBERGER, T. K. 1994. Random amplified polymorphic DNA in perennial ryegrass: a comparison of bulk samples vs. individuals. Hort. Sci., 29, 624-626.
WEIR, B. S. 1996. Genetic Data Analysis II. Sinauer Associates, Sunderland, MA.

YU, P. AND PAULS, K. P. 1993. Rapid estimation of genetic relatedness among heterogeneous populations of alfalfa by random amplification of pooled genomic DNA samples. Theor. Appl. Genet., 86, 788-794. 